\title{
Miniconference on astrophysical jets
}

\author{
P. M. Bellan \\ Applied Physics, California Institute of Technology, Pasadena, California 91125
}

(Received 24 January 2005; accepted 25 February 2005; published online 9 May 2005)

\begin{abstract}
This miniconference brought together observers of astrophysical jets, analytic and numerical modelers of both astrophysical jets and spheromaks, and laboratory experimentalists. The purpose of the miniconference was to encourage interaction between these diverse groups and also expose the plasma physics community to the interesting plasma issues associated with astrophysical jets. The miniconference emphasized magnetically driven astrophysical jets and consisted of three half-day sessions. The order of presentation was approximately: observations and general properties, experiments, numerical models, and special topics. (c) 2005 American Institute of Physics.
\end{abstract}

[DOI: $10.1063 / 1.1900563]$

\section{INTRODUCTION}

Astrophysical jets are long, narrow collimated structures observed to emanate from young stellar objects (YSO), black holes, and active galactic nuclei (AGN). The sizes and velocities of these jets vary over many orders of magnitude. YSO jets are typically nonrelativistic whereas black hole and AGN jets are relativistic. Until about 20 years ago it was generally believed that astrophysical jets were purely hydrodynamic, but since then it has been known that the acceleration mechanism for most jets is probably magnetohydrodynamic (MHD). While the jets are believed to be launched by some kind of MHD mechanism, at least in the case of YSO jets, it is possible that far from the star MHD ceases to be important in which case the jets become predominantly hydrodynamic.

Jets were initially considered a curiosity but now appear to be a fundamental aspect of star formation. Space-based telescopes such as the Hubble Space Telescope and the Spitzer Infrared Space Telescope have provided much higher resolution images than previously achieved and have even provided animated movies showing jet flow.

Outstanding questions regarding astrophysical jets include (i) the extent to which jets are magnetohydrodynamic or purely hydrodynamic, (ii) the mechanism for collimating jets, (iii) the role jets play in angular momentum transport in accreting compact objects and in star formation, (iv) the nature of knotlike structures observed in jets, and (v) interactions between jets and material impacted by the jet, such as molecular clouds.

Jets have been studied via direct observation, analytical models, and numerical models, and most recently by creating laboratory configurations which simulate important features of jets. One of these laboratory methods is based on spheromaks, a toroidal magnetic confinement configuration involving self-organization. Spheromaks have been studied for the last two decades and most recently, it has been realized that the physics of spheromak formation has much in common with magnetohydrodynamically driven astrophysical jets. Both spheromaks and proposed models of astrophysical jets involve an initial poloidal magnetic field which is then inflated outwards by a toroidal field associated with a poloidal current. Numerical models of spheromaks are quite similar to numerical models of astrophysical jets and so much can be learned by comparing spheromak physics to magnetohydrodynamically driven jet physics. Laboratory simulations of jets can also be produced by other means besides spheromak technology. In particular, high power pulse lasers can produce hydrodynamically driven jets and $Z$-pinch wire arrays can produce both hydrodynamically driven and magnetically driven jets.

This miniconference, organized by Bellan, brought together observers of astrophysical jets, analytic and numerical modelers of both astrophysical jets and spheromaks, and laboratory experimentalists. The purpose of the miniconference was to encourage interaction between these diverse groups and also expose the plasma physics community to the interesting plasma issues associated with astrophysical jets. The miniconference consisted of three half-day sessions. The order of presentation was approximately: observations and general properties, experiments, numerical models, and special topics. Co-authors of the talks are listed in the Bulletin of the American Physical Society for this conference; only the names of the presenter are given below.

\section{SESSION I}

Noriega-Crespo of the Spitzer Science Center at Caltech presented new observations of the Herbig-Haro (HH) 46/47 objects taken with the three instruments aboard the Spitzer Space Telescope, namely, the Infrared Array Camera (IRAC), the Multiband Imaging Photometer for Spitzer, and the Infrared Spectrograph. The optically invisible southwest lobe, driven by a jet which interacts with the surrounding medium creating what is known as the HH $47 \mathrm{C}$ bowshock, is revealed in full detail by the IRAC images, and displays a looplike morphology. The midinfrared outflow lobes correspond to warm gas and dust and are narrower than the lobes corresponding to $\mathrm{CO}$ flow which is a tracer of the cold molecular gas. The combination of emission by $\mathrm{H}_{2}$ rotational lines $[S(11)-S(4)]$ and some atomic lines, which fall within the IRAC passbands, are believed responsible for the bulk of the observed emission, although contributions from the 3.3, 6.2 , and $7.7 \mu \mathrm{m}$ polycyclic aromatic hydrocarbon emission 
bands cannot be ruled out. The limb-brightened cavity seen in the new Spitzer infrared images of the southwest lobe of HH 46/47 was modeled as the bow shock driven by an outflow from a young, low mass star. Purely hydrodynamic models in which the outflow is (i) a perfectly collimated, straight jet, (ii) a precessing jet, or (iii) a latitude-dependent wind were presented. Cases in which the outflow moves into a constant density cloud and into a stratified cloud were discussed and best agreement with the observed cavity was obtained for the precessing jet in a stratified cloud. However, the "straight jet" (traveling in a stratified cloud) also gives cavity shapes close to what is observed. The "latitudedependent wind" model gives cavity shapes substantially wider than what is observed.

Ray of the Dublin Institute for Advanced Studies showed that the birth of stars involves not only the formation of accretion disks but also the generation of highly collimated jets/outflows. These outflows can stretch for several parsecs, transporting energy and momentum to the surrounding cloud and even, in some cases, producing turbulent cloud support against gravity. While it is very obvious that magnetic fields play a significant role in the generation and collimation of AGN jets, their role in jets from YSOs is less obvious. This is primarily because good diagnostics for measuring field strength in YSO jets are still lacking although excellent probes exist for other basic parameters, e.g., velocity, density, temperature, etc. Some idea of the role of magnetic fields, however, can be gleaned indirectly from observations of rotation in a number of jets. These observations were made using the long-slit spectrograph on board the Hubble Space Telescope. The typical amount of rotation observed suggests that a significant fraction and possibly all of the angular momentum of the matter accreted through the disk is transported through the jet. This is exactly as predicted by magnetocentrifugal launching models. Ray noted that very rough estimates of magnetic field strengths can be derived from emission line ratios in the postshock cooling zones of jets. Finally in very rare cases, e.g., T Tauri South, gyrosynchrotron radiation has been observed and the field estimated from observations of circular polarization. This method may prove particularly useful in the future when a new generation of highly sensitive radio interferometers currently under construction become operational.

Bellan of Caltech described a spheromak-based laboratory plasma configuration ${ }^{1}$ in which many important features of magnetically driven astrophysical jets can be simulated. The experimental sequence starts with a quasistatic seed poloidal magnetic field which links a central disk electrode to a coplanar bounding annular electrode; this arrangement provides a topology analogous to the poloidal magnetic field of a star linking a surrounding accretion disk. After puffing neutral gas from nozzles mounted on the electrodes, plasma is created via application of a large emf between the central disk and the bounding annular electrode. The emf then drives a large poloidal electric current flowing from the central disk electrode (star) to the annulus (accretion disk) along the bias poloidal magnetic field. This electric current produces large magnetohydrodynamic forces which result in dynamics analogous to the dynamics of an astrophysical jet. In particu-

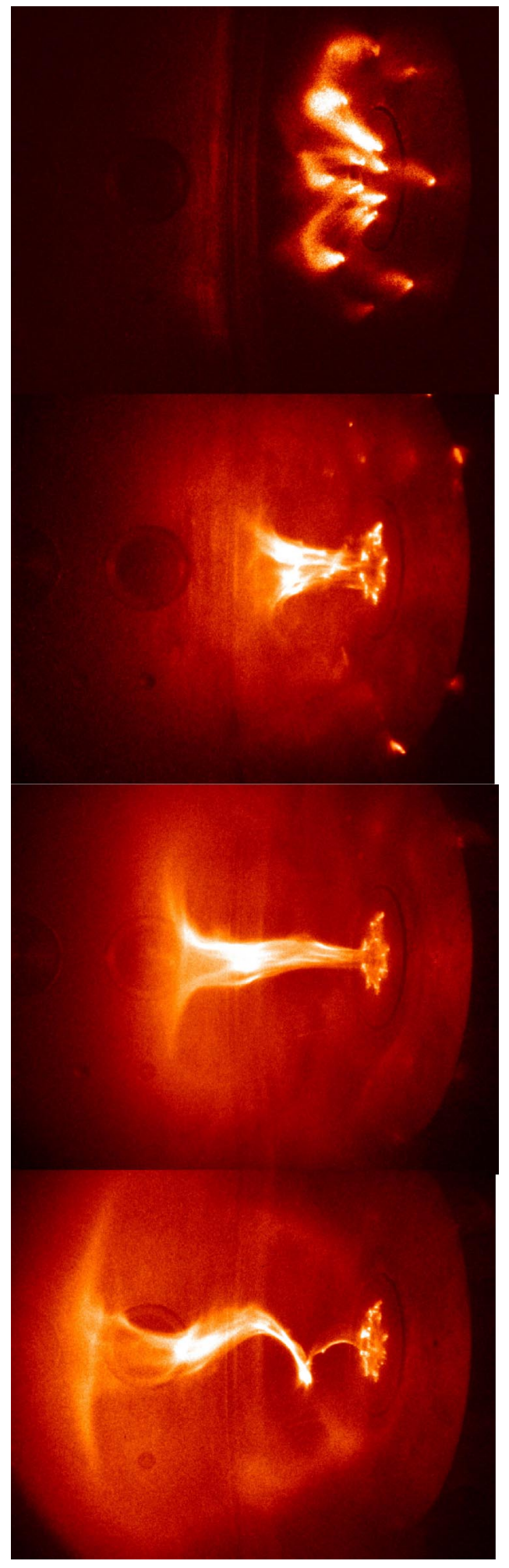

FIG. 1. Sequence of jet formation and kink instability in lab experiment (from Bellan's talk).

lar, the laboratory "astrophysical jet" is observed ${ }^{2-4}$ to evolve through a distinct, reproducible sequence consisting of jet formation, collimation, and kink instability. This sequence is shown in Fig. 1 from Refs. 2 and 3. For appropriate parameters, the kinked jet detaches and forms an unbounded, expanding spheromaklike plasmoid. These observations and re- 


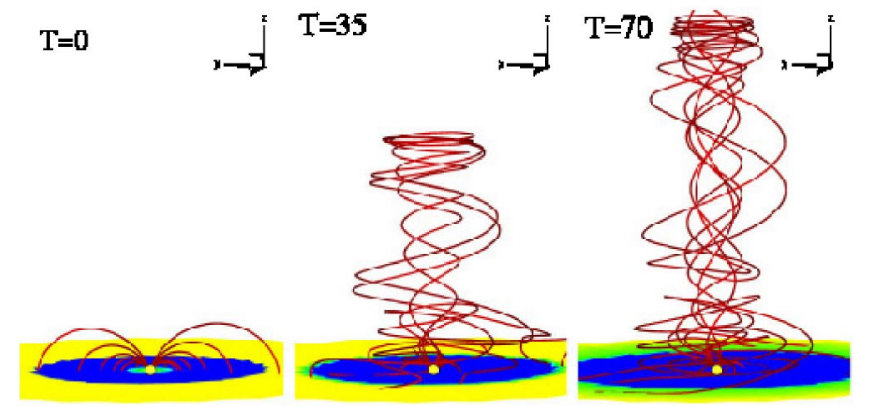

FIG. 2. Three-dimensional views of the temporal evolution of an MHD jet driven by the rapid rotation of a magnetized star (from Lovelace's talk).

lated observations on a solar prominence simulation experiment ${ }^{5}$ have motivated an analytic model ${ }^{6}$ for the collimation physics whereby stagnation of convected, frozen-in toroidal magnetic flux amplifies the toroidal magnetic flux density and then, since the toroidal magnetic field (i.e., toroidal flux density) provides the pinch force, the pinch force is increased, collimating the jet.

Lovelace briefly surveyed the many types of jets ranging from powerful radio, and in some cases optical and $\gamma$-ray emitting jets emanating from many compact accreting objects, from stellar mass black holes to supermassive black holes in galactic nuclei. The jets are widely thought to arise from the twisting of an ordered magnetic field threading a differentially rotating accretion disk which acts to magnetically extract angular momentum and energy from the disk. Two main regimes were distinguished: hydromagnetic jets, which have a significant mass flux and have energy and angular momentum carried by both matter and electromagnetic field and, Poynting jets, where the mass flux is small and energy and angular momentum are carried predominantly by the electromagnetic field. The energy flux is given by the familiar Poynting vector while the angular momentum flux is given by $\mathbf{r} \times \mathbf{T}_{\text {Max }}$ where $\mathbf{T}_{\text {Max }}$ is the Maxwell stress tensor. Recent MHD simulations show the formation of Poynting jets in the disk accretion to a rapidly rotating star with a dipole magnetic field - the propeller effect (Fig. 2, see Ref. 7). Recent theoretical work on the formation of Poynting jets from magnetized accretion disks was discussed ${ }^{8}$ and new relativistic, fully electromagnetic, particle-in-cell simulations of the formation of jets from accretion disks were presented (Fig. 3; see Ref. 9).

Pudritz of McMaster University noted that astrophysical jets are observed in a wide variety of systems ranging from stars in the act of formation to quasars. In most if not all of these systems, a central object such as a massive black hole or a young star accretes gas from a surrounding, Keplerian, gaseous disk. One of the most popular and best tested models for jet formation posits that jets are highly collimated, hydromagnetic winds that are flung out from the surfaces of accretion disks along open field lines that thread them. Basic theoretical calculations predict that these jets are powered by the gravitational potential energy that must be released as gas in the underlying disk slowly spirals in towards the central object. In addition, the magnetic torque exerted by such a wind can efficiently extract the angular momentum of gas

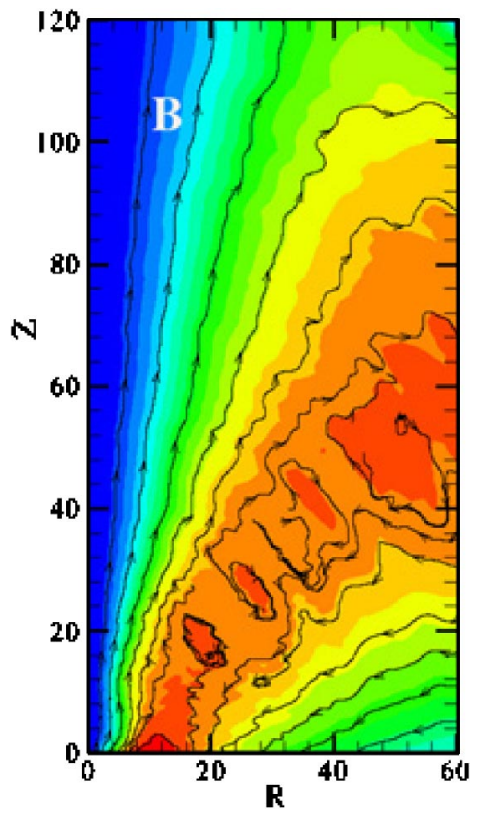

FIG. 3. Space charged limited emission from the disk causes the field to open and results in a steady Poynting flux outflow (from Lovelace's talk).

within the disk, enabling the accretion process. Recent observations of jets from young stellar objects reveal that these flows rotate and can be traced back to an extended region of the underlying disk, and these provide stringent tests for the model. The basic elements of this picture were reviewed and shown to provide a promising universal jet model which is consistent with sophisticated three-dimensional (3D) numerical simulations. ${ }^{10}$

Königl of the University of Chicago argued that magnetic fields should play a key role in the powering of astrophysical jets (through extraction of rotational kinetic energy from a compact central mass or its surrounding accretion disk) and in the acceleration and collimation of these outflows. Using semianalytic MHD models, the following issues were addressed:

(1) How are jets launched from accretion disks? An example of a steady-state protostellar ambipolar diffusiondominated disk from which hydromagnetic jets are launched along open magnetic field lines was presented ${ }^{11}$ and it was argued that the equilibrium solution curves in parameter space can be used to deduce that real disk/jet systems of this type are likely stable. ${ }^{12}$

(2) How do magnetic stresses accelerate and collimate the flow? Exact semianalytic solutions of the "hot" specialrelativistic MHD equations employing radial self-similarity ${ }^{13}$ were discussed as an example of the applicability of the MHD jet model to $\gamma$-ray burst sources, AGN, and other astrophysical systems where relativistic jets are observed. Although centrifugal and thermal driving may be important initially, the bulk of the acceleration of relativistic jets is due to the (toroidal) magnetic pressure-gradient force and is spatially extended (i.e., the scale lengths are much larger than the size of the source). This feature, also manifested to a lesser degree in nonrelativistic flows, is an identifying characteristic which distinguishes hydromagnetic acceleration 
from alternative driving forces. Recent observations of AGN have uncovered evidence for extended acceleration to relativistic speeds that is most naturally explained in this fashion. ${ }^{14}$ The helical trajectories often exhibited by synchrotron-emitting knots in relativistic AGN jets can be attributed to the motion of plasmoids along equilibrium helical magnetic field lines (no instability is invoked, in contrast with the interpretation outlined in Nakamura's presentation).

(3) How does the magnetic field advected by the flow influence the equilibrium structure of the jet beyond the acceleration region? The possibility that the magnetic field, which dominates the internal energy density far from the origin, settles into a minimum-energy Taylor state determined by the magnetic helicity and flux injected at the source and by the value of the confining ambient pressure, was outlined and illustrated by applications to AGN jets. ${ }^{15}$ It was pointed out that observations of jets such as NGC 6251 may make it possible to relate the properties of the smallscale magnetic acceleration region and the large-scale magnetic relaxation region in a single source.

\section{SESSION II}

Bally of the University of Colorado stated that most stars produce highly collimated jets during birth. Stellar jets have velocities ranging from less than 100 to over $1000 \mathrm{~km} / \mathrm{s}$, attain lengths of tens of light years, and play a major role in the generation of interstellar turbulence. ${ }^{16}$ Protostellar jets exhibit physical properties ranging from weakly ionized, mostly molecular, and dusty media to fully ionized plasmas. Most stellar jets are probably accelerated and collimated by MHD processes. Protostellar jets are ideal laboratories for the investigation of the launch, collimation, and termination of all types of astrophysical jet because radial velocities, proper motions, densities, and composition can be measured. Jet symmetries provide evidence for variability in mass-loss rate, velocity, orientation, and degree of collimation. Jets propagating through molecular clouds appear to be the drivers of several secondary markers of outflow phenomena from young stars such as shock-excited near-infrared $\mathrm{H}_{2}$ and Fe II emission and molecular outflows traced by millimeterwavelength emission lines such as CO. Partially or fully ionized jets usually trace the fastest portions of outflows. Shockexcited $\mathrm{H}_{2}$ and/or $\mathrm{Fe}$ II, which tend to exhibit lower velocities than the jet, trace the interfaces where these jets impact the surrounding medium. Less collimated lobes of $\mathrm{CO}$ emission trace the ambient molecular gas accelerated by this interaction and tend to be the lowest velocity but most massive component of YSO outflows.

Lapenta of Los Alamos National Lab discussed the creation of jets in active galactic nuclei (AGN). These jets are created in accretion disks around supermassive black holes and can retain their collimation up to the order of megaparsecs. Understanding the physical causes of this remarkable collimation over such large distances is a great challenge, and could lead to important new insights regarding the physics of magnetoplasmas. A two-pronged approach is used to attack this problem. First, the observational evidence from

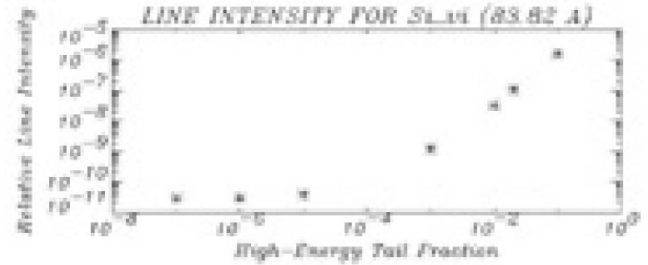

FIG. 4. Calculated enhancements of line ratios in $\mathrm{Si}$ in the presence of a hot electron tail (from Rose's talk).

two well-defined jets is gathered and organized. One of these jets, from the AGN of M87, is relatively small and close, and propagates within a galaxy cluster environment. The other jet is from the more distant radio galaxy $3 \mathrm{C} 303$, and has greater dimensions and luminosity. New constraints are derived for the plasma properties of both jets. Second, the behavior of these jets is simulated using a recently developed mathematical model. ${ }^{17}$ The goal is to estimate plasma parameters for the two jets and to determine the ability of MHD models to describe the collimation of extragalactic jets.

Rose of Mission Research Corporation noted that the innermost few parsecs of AGN jets show bulk relativistic particle motion. ${ }^{18}$ Magnetic self-compression has been invoked as one model for inductive acceleration of charged particles to relativistic energies. ${ }^{19}$ Such a collection of fast streaming electrons may easily acquire a momentum distribution characterized by moderately broad angle spread and rather narrow energy spread. ${ }^{20}$ The consequences of a relativistic electron beam passing through a denser, nonrelativistic plasma were discussed and various plasma wave mechanisms for coupling energy from the beam to the background plasma were considered so as to establish a nonthermal dissipative quasisteady state of the beam-penetrated plasma. ${ }^{21,22}$ This dynamical picture is fundamentally different from the MHD description of plasma heating by a penetrating beam. Plasma wave mechanisms under investigation included primary unstable space-charge waves transferring power to nonlinearly induced secondary space charge waves and density fluctuations (cavitons), and in which nonthermal randomly accelerated plasma electrons created by these nonlinearities provide and represent greatly enhanced Landau damping of the secondary waves. ${ }^{23}$ The power balance of the energized nonthermal hot electrons and the three wave populations are studied for such astrophysical applications, and used to allow some inference about beam-plasma properties from potential Bremsstrahlung and line spectrum observations. ${ }^{18,24}$ Figure 4 shows a sample calculation for line enhancements in $\mathrm{Si}$ in the presence of a hot plasma electron tail. These results are obtained from the XSTAR code, ${ }^{25}$ modified to treat nonthermal temperature distributions.

Lebedev of Imperial College presented experimental results on the formation of supersonic radiatively cooled plasma jets with dimensionless parameters (Mach number $\sim 20$, cooling parameter $\sim 1$, and density contrast $\rho_{j} / \rho_{a}$ $\sim 10$ ), similar to YSO jet parameters. Two different experimental configurations studied on the MAGPIE Z-pinch pulsed power facility were discussed. The jets in the first 
configuration $^{26,27}$ are purely hydrodynamic and used to study jet deflection by crosswinds, thus modeling the $\mathrm{C}$-shaped astrophysical YSO jets discussed by Bally above. The crosswind leads to jet deflection ${ }^{28}$ through formation of internal oblique shocks in the jet. Jets in the second set of experiments are driven by a toroidal magnetic field in a configuration $^{29}$ similar to astrophysical "magnetic tower jets." Experimental data show formation and axial inflation of laboratory magnetic tower jets, consisting of central current-carrying plasma column collimated by the hoop stress, which is surrounded by a magnetic cavity with the return current flowing along the walls of this cavity. The development of MHD $m=0$ and $m=1$ instabilities leading to the formation of the knots in the jet is clearly seen, but these instabilities do not destroy the jet. A modification of the experimental configuration to allow addition of a poloidal magnetic field and angular momentum in future experiments was also discussed. The experimental results were compared with computer simulations performed with laboratory plasma and astrophysical codes.

Tang of Los Alamos National Laboratory discussed how a conducting accretion disk in a plasma environment makes a natural dynamo machine that converts orbital mechanical energy into an expanding magnetic jet/lobe via magnetic helicity injection by differentially twisting a magnetic arcade line tied on the disk. In the laboratory, a radial electric field imposed by coaxial electrodes simulates the same process as described in the Caltech experiment. The magnetic morphology of a freely expanding jet/lobe is found to be dependent on the disk rotation speed $v_{r}$ with respect to the coronal Alfvén speed $v_{A}$. The collimation effect of the plasma inertia becomes most pronounced when $v_{r} \gg v_{A}$. In a dissipative plasma, axisymmetric steady state can be reached with magnetic flux $\propto v_{r}^{\alpha}(\alpha<1)$ and plasma $\mathbf{E} \times \mathbf{B}$ expansion speed $\propto_{v_{r}}$. A stagnated plasma lobe would become unstable as soon as the injected toroidal flux is greater than the poloidal flux (the so-called curse of the spheromak), and undergo flux conversion following a helical instability cascade. ${ }^{30}$ In a freely expanding plasma, rapid increase in the toroidal flux modifies the rotational transform profile of the open field lines quickly and hence avoids locking to a primary eigenmode that could destroy the jet morphology at early stage of the expansion. If the plasma is indeed force-free and eventually Taylor relaxed, Jensen-Chu resonance ${ }^{31}$ predicts a spheromak solution for the entire radio lobe. In a more realistic partially relaxed plasma, Jensen-Chu resonances are regularized and more complicated magnetic structures become accessible. ${ }^{32}$

You of Caltech presented direct measurements of the formation process of axially expanding, collimated, plasmafilled magnetic flux tubes in the Caltech experiment discussed in broad outline by Bellan. The results strongly support a recent MHD model ${ }^{6}$ explaining why currentcarrying magnetic flux tubes become collimated and dense. The process occurs in less than $1 \mu \mathrm{s}$ in the experiment and involves acceleration by MHD forces to achieve flow velocities of the order of $\sim 100 \mathrm{~km} / \mathrm{s}$. Densities of $10^{22} \mathrm{~m}^{-3}$ are achieved in the center column "astrophysical jet" ${ }^{\text {"2 }}$ that expands axially into a large vacuum chamber. Measurements

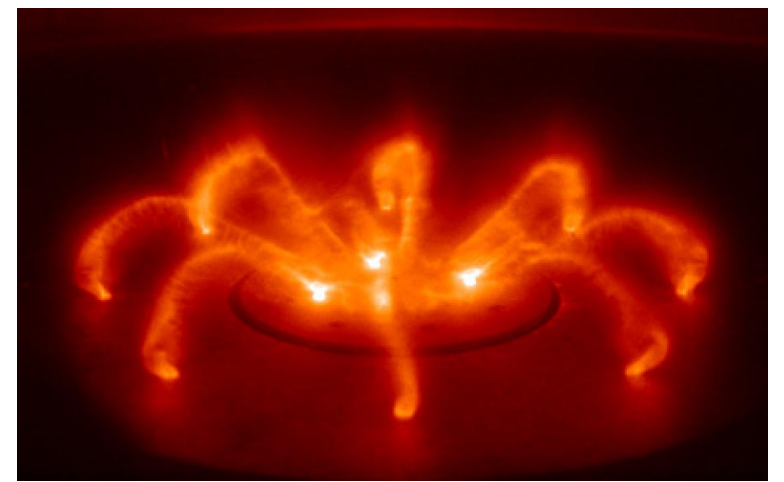

FIG. 5. Eight collimated flux tubes resembling "spider legs" (from You's talk).

show that the initial neutral gas cloud required for breakdown cannot account for such a large jet density and that plasma is ingested into magnetic flux tubes resembling "spider legs" on microsecond time scales by MHD pumping; a typical photo of these spider leg flux tubes is shown in Fig. 5. These observations resolve the sequence of steps leading to the collimation of current-carrying magnetic flux tubes.

\section{SESSION III}

Colgate of Los Alamos National Laboratory described a liquid sodium $\alpha-\Omega$ dynamo experiment constructed at New Mexico Institute of Mining and Technology to simulate magnetorotational instability, dynamo gain, and feedback in liquid sodium $\left(r_{1}=15 \mathrm{~cm}, r_{2}=30 \mathrm{~cm}, L=30 \mathrm{~cm}, f_{1}\right.$ $=120 \mathrm{~Hz}, f_{2}=30 \mathrm{~Hz}$ ). This experiment is designed to simulate the generation of large-scale magnetic fields in massive black-hole accretion disks, galaxies, and stars. The omega gain is due to the shear flow of differential rotation of Couette flow between two differentially rotating coaxial cylinders. Differential rotation in a conducting fluid twists a radial or quadrupole magnetic flux into a greatly enhanced toroidal flux. A large coherent helicity is produced by driven plumes and astrophysically by star-disk collisions, supernova explosions, or large-scale plume convection, respectively. The device has undergone preliminary tests using water and hot oil and has demonstrated stable Couette flow with only Ekmanflow-induced torque.

Blackman of the University of Rochester showed that coronae of accretion disks from which jets emerge, and stellar corona share much in common. Both represent magnetically active environments anchored in turbulent rotators below. The geometry of jet-mediating magnetic fields is analogous to that of stellar coronal holes. The question of where the fields of these jets come from is important. In the sun, sign reversals of the large-scale coronal hole fields imply the field is produced in situ, not simply accreted from the original material from which the sun formed. Such reversals cannot be measured in accretion disks, but the similarity between the two coronal environments and the difficulty of accreting large-scale fields in thin disks suggest a role for in situ formation. ${ }^{33}$ The physics behind a two-stage framework for understanding large-scale field formation in coronae was discussed: (1) a helical dynamo in the rotator supplies the 


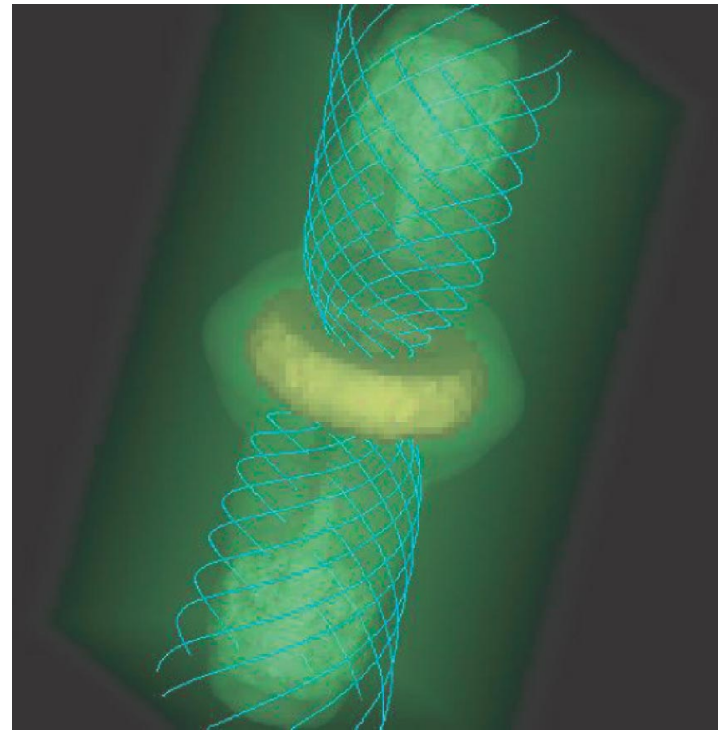

FIG. 6. A result of global resistive MHD simulation of jet formation from an accretion disk. Color scale shows density distribution. Solid curves show magnetic field lines (from Matsumoto's talk).

corona with bihelical structures of small net helicity, ${ }^{34}(2)$ bihelical magnetic relaxation in the corona grows global scale helical fields of a dominant sign. ${ }^{35}$

Li of Los Alamos National Laboratory stated that magnetic fields play an important role in determining the overall structures of astrophysical jets, especially in extra-galactic jets powered by supermassive black holes at centers of galaxies. Many questions remain regarding such objects, one of which is the mechanism(s) for collimation. This is a particularly difficult, multiscale problem since it involves the evolution of magnetic fields driven by the black-hole accretion disk engine, followed by the propagation through the galaxy and expansion into the general intergalactic medium. Three dimensional MHD simulations (both ideal and resistive) of the formation of large scale magnetic jets/"towers" were presented. These jets evolved from an idealized initial state where the "engine" is modeled as a highly wound and compressed magnetic "spring." A detailed analysis of the struc-
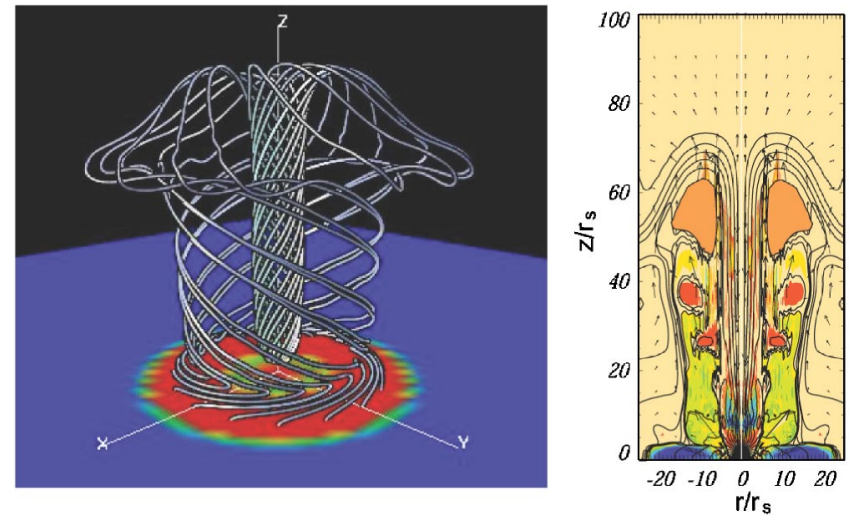

FIG. 7. Left: magnetic tower created by the expansion of magnetic loops twisted by the rotation of the disk; solid curves show magnetic field lines. Right: injection of hot plasmoids into the magnetic tower; solid curves show magnetic field lines, color scale (from Matsumoto's talk).
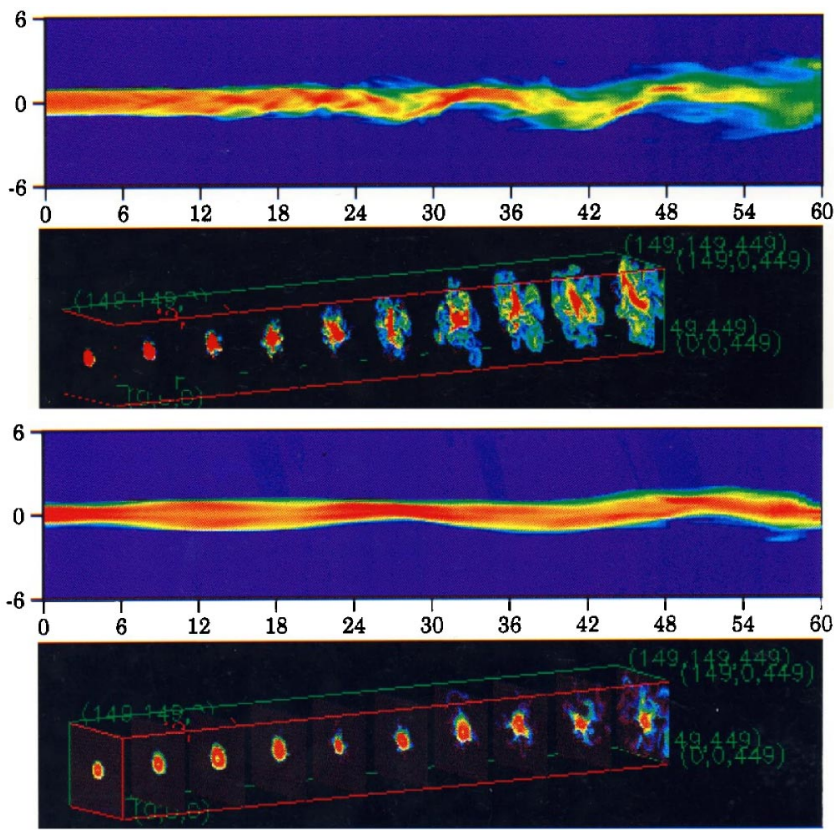

FIG. 8. Pseudosynchrotron intensity images and transverse slices of the magnetic pressure. The top image pair shows various helical structures along with the result of large-scale helical twisting for an equipartition poloidal magnetic field. The bottom image pair shows the stabilizing influence provided by an equipartition helical magnetic field (from Hardee's talk).

ture of the "tower" showed that magnetic fields are selfcollimated by their pinch forces and maintain stability by rapidly reducing the toroidal to poloidal flux ratio. The front end of the propagating jet, however, undergoes the 3D kink instability, converting toroidal fluxes to poloidal fluxes. This might have interesting applications to the formation of the giant radio lobes observed in extra-galactic radio sources.

Matsumoto of Chiba University presented results of global numerical simulations of magnetically driven jets associated with accretion disks. The accretion disk was included in the computational region so that the back reaction of the jet formation on the disk is incorporated. Magnetically driven jets can be classified into magnetocentrifugally driven jets and magnetic tower jets. The former is a heavy jet in which disk material is accelerated. The latter is a light jet dominated by Poynting flux. When the disk is threaded by global open magnetic fields, magnetocentrifugal force drives the outflow. Time-dependent, MHD simulations of jet formation from an accretion disk were first carried out by Uchida and Shibata. ${ }^{36,37}$ A frequently asked question for such simulations is whether steady outflows can be obtained. Resistive MHD simulations indicate that magnetorotational instability makes the outflows intermittent when resistivity is small but outflows from mildly resistive disks become quasisteady. ${ }^{38}$ Figure 6 shows the distribution of density and magnetic field lines obtained by such mildly resistive simulation. The acceleration mechanism of the resistive jet is also the magnetocentrifugal force. When magnetic fields connect the central star and its accretion disk, a magnetic tower jet is formed. Twist injection from the disk or from the star inflates the magnetic loops connecting the star and the disk; magnetic reconnection takes place in the current sheet created inside 

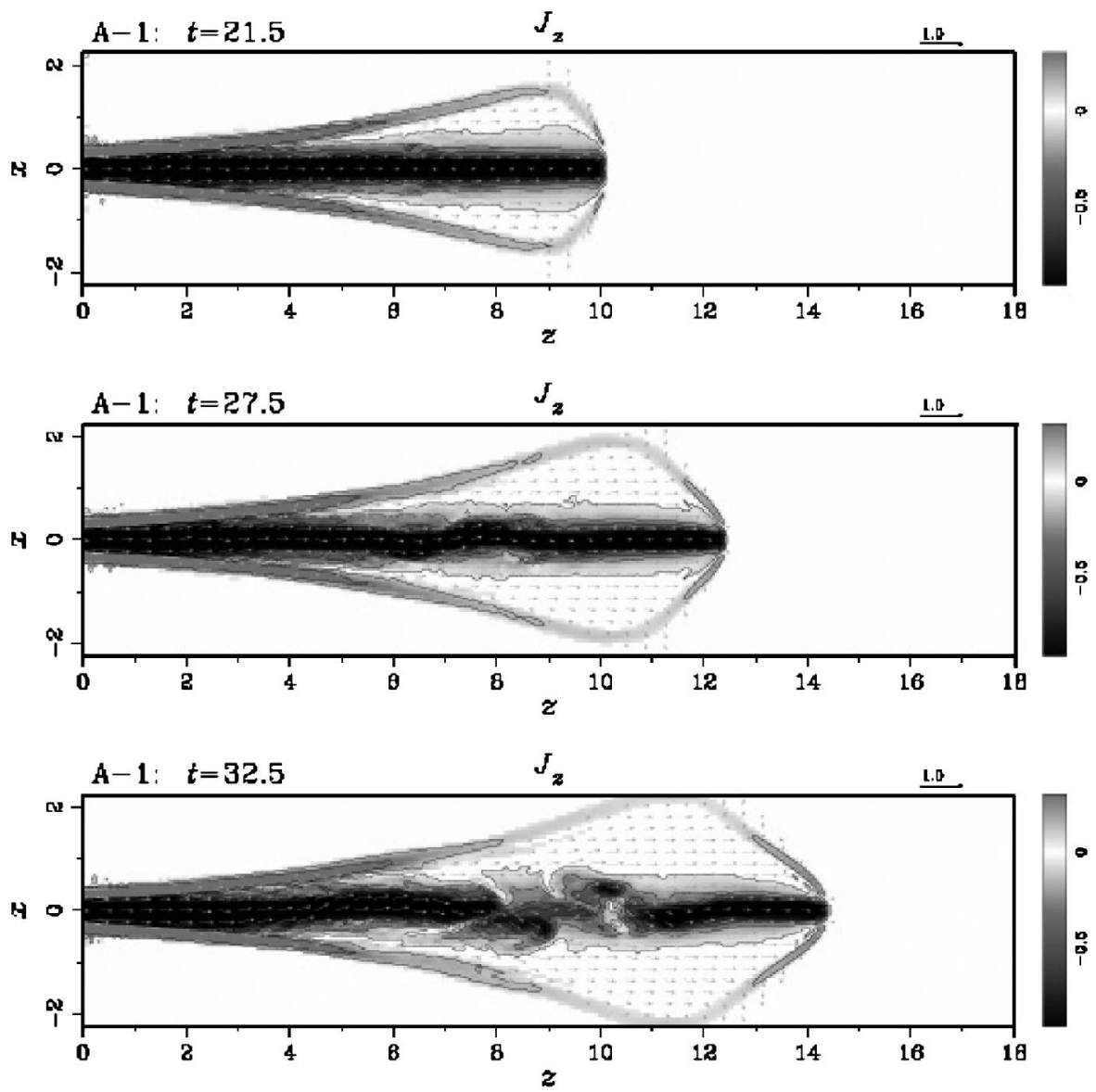

FIG. 9. Time evolution for a typical Poynting flux-dominated jet model (Nakamura and Meier, 2004). Contour images of the axial current density $J_{z}$ are shown along with the poloidal velocity field in the $x-z$ plane. The figures show a closed circulating current system, in which one current path occurs near the central $(z)$ axis and comoves with the PFD jet- "jet current," while the other conically shaped current returns outside- "return current." The actual jet structures in this model are a three-dimensional spiral helix.

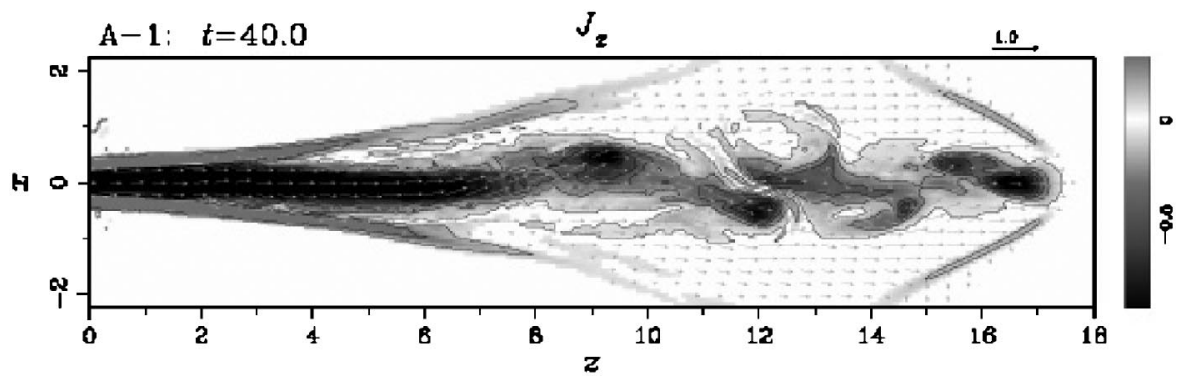

the expanding magnetic loops. ${ }^{39}$ When external gas pressure is large enough, the expanding loops form a magnetic tower, in which Poynting flux dominates the energy transport (Fig. 7). This mechanism can explain the formation of relativistic jets in $\mathrm{x}$-ray binaries containing a neutron star. ${ }^{40}$ The results of global three-dimensional simulations of state transitions in black-hole accretion flows were presented. As the disk makes transition from a low/hard (hot) state to a high/soft (cool) state, a disk dominated by magnetic pressure is created. Release of the magnetic energy can drive relativistic outflows dominated by Poynting flux.

Hardee of the University of Alabama reviewed some of the basic theoretical stability properties of jets. A likely scenario is that jets are magnetically dominated in the acceleration and collimation regions but are kinetically dominated at large scales. In both the magnetic and kinetic regimes the CD (current-driven) helical kink (see Bellan; Nakamura in this summary paper) and KH (Kelvin-Helmholtz) driven helical surface modes, respectively, are dominant and potentially the most disruptive to highly collimated flow. Clearly, astrophysical jets are sufficiently stabilized, either linearly or nonlinearly, to these modes. In the kinetic regime a combination of high Lorentz factor and/or magnetosonic Mach number, jet expansion, velocity shear, and magnetic field configuration can serve to partially stabilize a jet (see Ref. 41). The importance of the magnetic configuration to partial stabilization in the kinetically dominated regime is illustrated by numerical simulation images in Fig. 8.

Similar partial stabilization effects must occur where astrophysical jets are in the magnetic regime (see Tang in this summary paper). Some examples of twisted astrophysical jet structures indicate that jets exhibit observable structures associated with the kink or helical modes. The CD kink mode will move with the flow. Thus, observed kink structures in the magnetically dominated regime would indicate jet speed and acceleration produced by the magnetic driving investigated by Vlahakis and Königl. ${ }^{14}$ The KH helical mode moves slower than the flow with speed a function of jet and 
external sound speeds and frequency relative to the most unstable frequency and can be used to estimate sound speeds and flow acceleration. ${ }^{42}$ The identification of observed jet structures with experimentally determined, numerically determined or theoretically determined structures may provide estimates of otherwise unobservable astrophysical jet properties.

Nakamura of the Jet Propulsion Laboratory argued that the spatial stability properties of astrophysical jets are one of the most important issues in jet dynamics. Nonrelativistic three-dimensional MHD simulations of Poynting flux dominated (PFD) jets were presented. The study of these jets focused on the propagation of strongly magnetized hypersonic, but sub-Alfvénic flow and on the subsequent development of a current-driven kink instability. This instability may be responsible for the "wiggled" structures seen in subparsec scale AGN jets and pulsar jets. Numerical results show that the Poynting flux dominated jets can develop current-driven distortions in the trans-Alfvénic flow case. An internal nonaxisymmetric body mode grows on time scales of order of the Alfvén crossing time and distorts the structure and magnetic configuration of the jet. The kink $(m=1)$ mode of the current-driven instability, driven by the asymmetrically distribution of hoop stress, grows faster than other higher order modes $(m>1)$ as shown in Fig. 9. This could be caused by a sudden loss of kinetic angular momentum to the magnetic field via the reverse slow-mode MHD shock wave. In the jet frame the mode grows locally and expands radially at each axial position where the jet is unstable: the instability, therefore, does not propagate as a wave along the jet length. The wiggled structures saturate and advect with the bulk flow and then, the local plasma flow follows a helical path along the kinked magnetic field backbone. ${ }^{43}$

Ryutov of the Lawrence Livermore National Laboratory argued that finite photon mass is compatible with general principles of relativity theory but the exact, small size of the photon mass must be established experimentally. The presently accepted upper limit ${ }^{44}$ is $10^{-22} m_{e}$ and has been estimated ${ }^{45}$ using solar wind observations. This estimate corresponds to a photon Compton length of $L=3 \times 10^{6} \mathrm{~km}$. This estimate could be improved upon by exploiting astrophysical jet properties, especially the pinch force which establishes the jet structure. It turns out that, if the jet radius is much greater than $L$, both pinch equilibrium and stability for a finite-mass photon become very different from the massless photon case. In particular, the equilibrium pressure maximum coincides with the maximum of the current density. Determining the extent to which these new features are incompatible with the observations provides a method for improving the estimate of the photon mass by orders of magnitude.

\section{ACKNOWLEDGMENTS}

This Astrophysical Jet Miniconference was sponsored by the American Physical Society Topical Group in Plasma As- trophysics and was supported in part by the U. S. Department of Energy and by the California Institute of Technology.

${ }^{1}$ P. M. Bellan, Spheromaks (Imperial College Press, London, 2000).

${ }^{2}$ S. C. Hsu and P. M. Bellan, Mon. Not. R. Astron. Soc. 334, 257 (2002).

${ }^{3}$ S. C. Hsu and P. M. Bellan, Phys. Rev. Lett. 90, 215002 (2003).

${ }^{4}$ S. C. Hsu and P. M. Bellan, Phys. Plasmas 12, 032103 (2005).

${ }^{5}$ J. F. Hansen and P. M. Bellan, Astrophys. J. 563, L183 (2001).

${ }^{6}$ P. M. Bellan, Phys. Plasmas 10, 1999 (2003).

${ }^{7}$ M. M. Romanova et al., Astrophys. J. Lett. 616, L151 (2004).

${ }^{8}$ R. V. E. Lovelace and M. M. Romanova, Astrophys. J. Lett. 596, L159 (2003).

${ }^{9}$ R. V. E. Lovelace, P. R. Gandhi, and M. M. Romanova, "Relativistic jets from accretion disks," Astrophys. Space Sci. (in press).

${ }^{10}$ R. Ouyed, D. A. Clarke, and R. E. Pudritz, Astrophys. J. 582, 292 (2003).

${ }^{11}$ M. Wardle and A. Königl, Astrophys. J. 410, 218 (1993).

${ }^{12}$ A. Königl, Astrophys. J. 617, 1267 (2004).

${ }^{13}$ N. Vlahakis and A. Königl, Astrophys. J. 596, 1080 (2003).

${ }^{14}$ N. Vlahakis and A. Königl, Astrophys. J. 605, 656 (2004).

${ }^{15}$ A. Königl and A. R. Choudhuri, Astrophys. J. 289, 173 (1985).

${ }^{16}$ B. Reipurth and J. Bally, Annu. Rev. Astron. Astrophys. 39, 403 (2001).

${ }^{17}$ G. Lapenta, Phys. Rev. Lett. 90, 135005 (2003).

${ }^{18}$ J. H. Beall, J. Guillory, D. V. Rose, S. Schindler, and S. Colafrancesco, "AGN Jet interactions with the intracluster medium," Chin. J. Astron. Astrophys. (in press).

${ }^{19}$ V. Semenov, S. Dyadechkin, and B. Punsly, Science 305, 978 (2004).

${ }^{20}$ L. E. Thode, Phys. Fluids 19, 831 (1976).

${ }^{21}$ W. K. Rose, J. Guillory, J. H. Beall, and S. Kainer, Astrophys. J. 280, 550 (1984).

${ }^{22}$ D. V. Rose, J. U. Guillory, and J. H. Beall, Phys. Plasmas 9, 1000 (2002).

${ }^{23}$ D. V. Rose, J. Guillory, and J. H. Beall, Phys. Plasmas 12, 014501 (2005).

${ }^{24}$ J. H. Beall, J. Guillory, and D. V. Rose, Mem. Soc. Astron. Ital. 70, 1235 (1999).

${ }^{25}$ T. R. Kallman and M. A. Bautista, Astrophys. J., Suppl. Ser. 133, 221 (2001).

${ }^{26}$ S. V. Lebedev, J. P. Chittenden, F. N. Beg, S. N. Bland, A. Ciardi, D. Ampleford, S. Hughes, M. G. Haines, A. Frank, E. G. Blackman, and T. Gardiner, Astrophys. J. 564, 113 (2002).

${ }^{27}$ A. Ciardi, S. V. Lebedev, J. P. Chittenden, and S. Bland, Laser Part. Beams 20, 255 (2002).

${ }^{28}$ S. V. Lebedev, D. Ampleford, A. Ciardi, S. N. Bland, J. P. Chittenden, M. G. Haines, A. Frank, E. G. Blackman, and A. Cunningham, Astrophys. J. 616, 988 (2004).

${ }^{29}$ A. Ciardi, S. V. Lebedev, J. P. Chittenden, D. J. Ampleford, S. N. Bland, S. C. Bott, and J. Rapley, "Modeling magnetic tower jets in the laboratory," Astrophys. Space Sci. (in press).

${ }^{30}$ X. Z. Tang and A. H. Boozer, Phys. Plasmas 11, 2679 (2004).

${ }^{31}$ T. H. Jensen and M. S. Chu, Phys. Fluids 27, 2881 (1984).

${ }^{32} \mathrm{X}$. Z. Tang and A. H. Boozer, "Force-free magnetic relaxation in driven plasmas," Phys. Rev. Lett. (submitted).

${ }^{33}$ E. G. Blackman and J. C. Tan, Astrophys. Space Sci. 292, 395 (2004).

${ }^{34}$ E. G. Blackman and G. B. Field, Phys. Rev. Lett. 89, 265007 (2002).

${ }^{35}$ E. G. Blackman, Phys. Plasmas 12, 1 (2005).

${ }^{36}$ Y. Uchida and K. Shibata, Publ. Astron. Soc. Jpn. 37, 515 (1985).

${ }^{37}$ K. Shibata and Y. Uchida, Publ. Astron. Soc. Jpn. 38, 631 (1986).

${ }^{38}$ T. Kuwabara, K. Shibata, T. Kudoh, and R. Matsumoto, Publ. Astron. Soc. Jpn. 52, 1109 (2000).

${ }^{39}$ M. R. Hayashi, K. Shibata, and R. Matsumoto, Astrophys. J. Lett. 468, L37 (1996).

${ }^{40}$ K. Kato, M. R. Hayashi, and R. Matsumoto, Astrophys. J. 600, 338 (2004).

${ }^{41}$ P. E. Hardee, Astrophys. Space Sci. 293, 117 (2004).

${ }^{42}$ P. E. Hardee, R. C. Walker, and J. L. Gómez, Astrophys. J. 620, 646 (2005).

${ }^{43}$ M. Nakamura and D. L. Meier, Astrophys. J. 617, 123 (2004).

${ }^{44}$ S. Eidelman, K. G. Hayes, K. A. Olive et al., Phys. Lett. B 592, 1 (2004).

${ }^{45}$ D. D. Ryutov, Plasma Phys. Controlled Fusion 39, A73 (1997). 